\title{
Leader-following consensus for fractional multi-agent systems
}

\author{
Ricardo Almeida ${ }^{1}$, Ewa Girejko², Snezhana Hristova ${ }^{3}$ and Agnieszka B. Malinowska ${ }^{2 *}$ (D)
}

${ }^{*}$ Correspondence:

a.malinowska@pb.edu.pl

${ }^{2}$ Faculty of Computer Science,

Bialystok, University of Technology,

Białystok, Poland

Full list of author information is

available at the end of the article

\begin{abstract}
A leader-following consensus for Caputo fractional multi-agent systems with nonlinear intrinsic dynamics is investigated. The second Lyapunov method is used to design a control protocol ensuring a consensus for two types of multi-agent systems. Contrary to the previous studies on leader-following consensus, the investigation covers systems with bounded and unbounded time-dependent Lipschitz coefficients in the intrinsic dynamics. Moreover, coupling strength describing the interactions between agents is considered to be a function of time.
\end{abstract}

Keywords: Multi-agent networks; Nonlinear fractional neural systems; Lyapunov function; Stability

\section{Introduction}

A canonical problem that appears in the coordination of dynamic multi-agent networks is the consensus problem: given initial values of nodes (agents), establish conditions under which, through local interactions and computations, nodes (agents) asymptotically reach an agreement upon a common state. The consensus problem plays an important role in various contexts such as wireless communication networks, sensor networks, leader election, clock phase synchronization, and air traffic control systems. It has been extensively studied by numerous researchers from different perspectives. There already exists a vast literature concerning first-order multi-agent systems [1-4], second-order multi-agent systems [5-7], fractional-order multi-agent systems [8-10], and fractional-order multiagent systems with a leader [11-14]. This paper is concerned with the last-mentioned case of systems, that is, we study a leader-following consensus for fractional multi-agent systems. Fractional calculus is a generalization of differentiation and integration to the arbitrary (non-integer) order. Fractional operators are excellent tools for modeling the memory-dependent phenomena $[15,16]$. Moreover, it was shown in [17] that fractionalorder derivative provides single neurons with a fundamental and general computation that can contribute to efficient information processing. Presently, when computations become faster and memory becomes cheaper, the application of fractional-order models is possible and affordable. Therefore, the stability properties of fractional-order neural networks are extensively studied by many researchers, see, e.g., [18-22] and the references therein. Many of real systems have been successfully described by models with fractional derivatives (see, for example, viscoelastic polymers [23], semi-infinite transmission lines with 
losses [24], dielectric polarization [25]). In engineering, the digital fractional-order controller was designed to control temperature in [26], the fractional-order PID controller was used in [27] to control the trajectory of the flight path. For a recent account of applications of fractional calculus in science and engineering, we refer the reader to [28].

In this paper, we consider a fractional-order system consisting of $\mathrm{N}$ agents, plus one additional agent, which acts as the group leader and which is independent of all other agents (followers). This means the agents have no influence on the dynamics of the leader. All agents and the leader share the same intrinsic dynamics given by a nonlinear function. In the literature, results concerning problems with intrinsic nonlinear dynamics have been reported, for example, in [11, 13, 14, 29, 30]. In [29, 30], the authors consider the fixedtime group consensus problems in networks of dynamic agents with unknown inherent nonlinear dynamics and with intrinsic nonlinear dynamics and bounded uncertainties, respectively. Systems are described by ordinary differential equations, and the results are obtained using Lyapunov theory, algebraic graph theory, and fixed-time stability. Systems more similar to the one considered in this paper, that is, fractional-order multi-agent systems, are investigated in $[11,13,14]$. The consensus control law is designed based on optimal control theory or algebraic graph theory and Lyapunov method. However, there are two main differences with respect to our framework: in $[11,13,14]$ the Lipschitz coefficient is a constant, while here we deal with the time-dependent Lipschitz coefficient. The second difference is that we do not assume a constant coupling strength in the interactions between agents. In our formulation, the coupling strength is a function of time. The main advantage of the proposed approach is that we can analyze a more general case, which covers a wider class of systems comparing with the ones that have already been studied in the literature. Moreover, it is worth to stress out that the coupling strength that we consider as a function of time (non-constant) is more natural and realistic for applications.

The contribution of this paper is summarized as follows. By the Lyapunov function method, we design a control law that guarantees the leader-following consensus: the state of each follower asymptotically converges to the state of the leader. Both cases of bounded Lipschitz coefficient and unbounded Lipschitz coefficient are studied. For any of these cases, we propose a set of sufficient conditions to guarantee a consensus. Compared with previous works, the major difficulty of the problem considered here is caused by the intrinsic nonlinear dynamics and is connected with its time-dependent Lipschitz coefficient and the time-varying coefficients in the control protocol. It requires the application of non-quadratic Lyapunov functions and their fractional derivatives different from the Caputo derivative. To tackle this difficulty, an appropriate extension of the Lyapunov second method is used.

The paper is organized in the following manner. Preliminaries section includes definitions, lemmas, and remarks used in the sequel. The next section contains our main results. After the introduction of the system of the model, the control protocol is proposed. Sufficient conditions for the leader-following consensus are provided in two subsections with two separate theorems. Theorem 1 concerns the case of multi-agent systems with intrinsic nonlinear dynamics described by a function with a bounded time-dependent Lipschitz coefficient and time-varying coefficients in the control protocol. The most general case is considered in Theorem 2. Namely, the function appearing in the intrinsic nonlinear dynamics has an unbounded time-dependent Lipschitz coefficient and coefficients in the 
control protocol are time-varying. In both cases, examples verifying theoretical discussion are provided.

\section{Preliminaries}

In this section, we recall the necessary definitions and facts that will be used in the sequel. Throughout the paper we follow the notation: $L_{1}([0, T))$ denotes the space of functions defined on $[0, T)$ for which the absolute value is Lebesgue integrable; $\mathrm{AC}([0, T))$ denotes the space of functions defined on $[0, T)$ that are absolutely continuous; $C([0, T))$ denotes the space of functions defined on $[0, T)$ that are continuous.

The Riemann-Liouville fractional derivative of order $q \in(0,1)$ of function $m \in$ $L_{1}([0, T)), T \leq \infty$, is given by (see, e.g., [31])

$$
{ }_{0}^{\mathrm{RL}} D_{t}^{q} m(t)=\frac{1}{\Gamma(1-q)} \frac{d}{d t} \int_{0}^{t}(t-s)^{-q} m(s) d s, \quad t \in[0, T),
$$

where $\Gamma(z):=\int_{0}^{\infty} t^{z-1} \exp (-t) d t, z>0$, is the gamma function.

The Caputo fractional derivative of order $q \in(0,1)$ of function $m \in \operatorname{AC}([0, T)), T \leq \infty$, is defined by (see, e.g., [31])

$$
{ }_{0}^{C} D_{t}^{q} m(t)=\frac{1}{\Gamma(1-q)} \int_{0}^{t}(t-s)^{-q} m^{\prime}(s) d s, \quad t \in[0, T) .
$$

Let us consider the $n$-dimensional Caputo fractional-order system

$$
{ }_{0}^{C} D_{t}^{q} x(t)=f(t, x(t)) \quad \text { for } t \in[0, T), \quad x(0)=x_{0},
$$

where $x:[0, T) \rightarrow \mathbb{R}^{n}, f \in C\left([0, T) \times \mathbb{R}^{n}, \mathbb{R}^{n}\right), T \leq \infty$, and $f(t, 0) \equiv 0, t \geq 0$. Observe that $\bar{x}=0$ is an equilibrium point of system (1), that is, ${ }_{0}^{C} D_{t}^{q} \bar{x}=0=f(t, \bar{x})$.

Definition 1 ([32]) An equilibrium point $\bar{x}=0$ of system (1) is asymptotically stable if, for any initial condition $x_{0}$, a solution to (1) satisfies $\lim _{t \rightarrow \infty}\|x(t)\|=0$, where $\|\cdot\|$ denotes an arbitrary norm in $\mathbb{R}^{n}$.

Similarly to integer-order systems, the Lyapunov second method can be used to analyze the asymptotic stability of nonlinear fractional-order system (1) without explicitly solving it. The main idea is to find an appropriate Lyapunov function for system (1). To this end, let $V$ be a Lyapunov function, i.e., $V:[0, T) \times \Delta \rightarrow \mathbb{R}_{+}$(where $\Delta \subset \mathbb{R}^{n}, 0 \in \Delta$ ) is continuous on $[0, T) \times \Delta$ and it is locally Lipschitz with respect to its second argument. In the literature, one can find mainly three types of derivatives of a Lyapunov function along the trajectories of the solutions of system (1).

1. The Caputo fractional derivative of $V$. Let $x:[0, T) \rightarrow \Delta$ be a solution of (1). Then

$$
{ }_{0}^{C} D_{t}^{q} V(t, x(t))=\frac{1}{\Gamma(1-q)} \int_{0}^{t}(t-s)^{-q} \frac{d}{d s}(V(s, x(s))) d s, \quad t \in[0, T) .
$$

This type of derivative is applicable for continuously differentiable Lyapunov functions. It is mainly used for quadratic Lyapunov functions to study several stability properties of fractional differential equations (see, for example, [33]). In the context 
of the Caputo fractional derivative of a Lyapunov function, the following lemma is useful.

Lemma 1 ([32]) Let $P \in \mathbb{R}^{n \times n}$ be a constant, symmetric, and positive definite matrix and $x: \mathbb{R}_{+} \rightarrow \mathbb{R}^{n}$ be a function for which the Caputo fractional derivative exists. Then

$$
{ }_{2}^{1}{ }_{0}^{C} D_{t}^{q}\left(x^{T}(t) P x(t)\right) \leq x^{T}(t) P_{0}^{C} D_{t}^{q} x(t), \quad t \in[0, T) .
$$

2. The Dini fractional derivative of $V$ (see [34]). Let $t \in[0, T)$ and $x \in \Delta$. Then

$$
\mathcal{D}_{(1)}^{+} V(t, x)=\limsup _{h \rightarrow 0} \frac{1}{h^{q}}\left[V(t, x)-\sum_{r=1}^{\left[\frac{t}{h}\right]}(-1)^{r+1}{ }_{q} C_{r} V\left(t-r h, x-h^{q} f(t, x)\right)\right],
$$

where ${ }_{q} C_{r}=\frac{q(q-1)(q-2) \ldots(q-r+1)}{r !}$.

Let us note that Dini derivative (3) is closer to both the Grunwald-Letnikov and the Riemann-Liouville fractional derivatives than to the Caputo fractional derivative. It does not depend on the initial value $V\left(0, x_{0}\right)$, which is typical for the Caputo derivative. It is applicable for continuous Lyapunov functions.

Remark 1 In the general case one has $\mathcal{D}_{(1)}^{+} V(t, x(t)) \neq{ }_{0}^{C} D_{t}^{q} V(t, x(t))$, where $x:[0, T) \rightarrow \Delta$ is a solution of (1).

3. The Caputo fractional Dini derivative of $V$ (see [34]). Let $t \in[0, T)$ and $x, x_{0} \in \Delta$, where $x_{0}$ is the initial condition of (1). Then

$$
\begin{aligned}
\underset{(1)}{C} D_{+}^{q} V\left(t, x ; 0, x_{0}\right)= & \limsup _{h \rightarrow 0^{+}} \frac{1}{h^{q}}\left\{V(t, x)-V\left(0, x_{0}\right)\right. \\
& \left.-\sum_{r=1}^{\left[\frac{t}{h}\right]}(-1)^{r+1}{ }_{q} C_{r}\left(V\left(t-r h, x-h^{q} f(t, x)\right)-V\left(0, x_{0}\right)\right)\right\}
\end{aligned}
$$

or using the equality ${ }_{0}^{\mathrm{RL}} D_{t}^{q} 1=\frac{1}{\Gamma(1-q)} t^{-q}=\lim \sup _{h \rightarrow 0^{+}} \frac{1}{h^{q}} \sum_{r=0}^{\left[\frac{t}{h}\right]}(-1)^{r}{ }_{q} C_{r}$ (see [31]) we obtain the equivalent form

$$
\begin{aligned}
\underset{(1)}{C} D_{+}^{q} V\left(t, x ; 0, x_{0}\right) \\
=\limsup _{h \rightarrow 0^{+}} \frac{1}{h^{q}}\left\{V(t, x)+\sum_{r=1}^{\left[\frac{t}{h}\right]}(-1)^{r}{ }_{q} C_{r} V\left(t-r h, x-h^{q} f(t, x)\right)\right\} \\
\quad-V\left(0, x_{0}\right) \limsup _{h \rightarrow 0^{+}} \frac{1}{h^{q}} \sum_{r=0}^{\left[\frac{t}{h}\right]}(-1)^{r}{ }_{q} C_{r} \\
=\limsup _{h \rightarrow 0^{+}} \frac{1}{h^{q}}\left\{V(t, x)+\sum_{r=1}^{\left[\frac{t}{h}\right]}(-1)^{r}{ }_{q} C_{r} V\left(t-r h, x-h^{q} f(t, x)\right)\right\}-\frac{V\left(0, x_{0}\right)}{t^{q} \Gamma(1-q)} .
\end{aligned}
$$


Let us observe that derivative (4) depends significantly on both the fractional-order $q$ and the initial data $\left(0, x_{0}\right)$ of system (1). This type of derivative is close to the idea of the Caputo fractional derivative. It is applicable for continuous Lyapunov functions.

Remark 2 For any $t>0$ and $x, x_{0} \in \Delta$, one has:

$$
\begin{aligned}
& \underset{(1)}{C} D_{+}^{q} V\left(t, x ; 0, x_{0}\right)=\mathcal{D}_{(1)}^{+} V(t, x)-\frac{V\left(0, x_{0}\right)}{t^{q} \Gamma(1-q)}, \\
& \underset{(1)}{C} D_{+}^{q} V\left(t, x ; 0, x_{0}\right)=\mathcal{D}_{(1)}^{+} V(t, x), \quad \text { if } V\left(0, x_{0}\right)=0, \\
& \underset{(1)}{C} D_{+}^{q} V\left(t, x ; 0, x_{0}\right)<\mathcal{D}_{(1)}^{+} V(t, x), \quad \text { if } V\left(0, x_{0}\right)>0 .
\end{aligned}
$$

In the next section, to prove the main results of the paper, we will use the following lemmas.

Lemma 2 (Theorem 11 from [35]) Let $\bar{x}=0$ be an equilibrium point for the nonautonomous fractional-order system (1). Assume that there exists a Lyapunov function $V(t, x)$ such that

$$
\begin{aligned}
& \alpha_{1}(\|x\|) \leq V(t, x) \leq \alpha_{2}(\|x\|), \quad t \geq 0, \quad x \in \Delta, \\
& { }_{0}^{C} D_{t}^{\beta} V(t, x(t)) \leq-\alpha_{3}(\|x\|), \quad t>0,
\end{aligned}
$$

where $\beta \in(0,1)$, functions $\alpha_{i} \in C([0, \infty),[0, \infty)), i=1,2,3$, are strictly increasing and $\alpha_{i}(0)=0$. Then the equilibrium point $\bar{x}=0$ of system (1) is asymptotically stable.

Lemma 3 (Theorem 3 from [36]) Let there exists the Lyapunov function $V$ such that $V(t, 0)=0, t \geq 0$, and

$$
\begin{aligned}
& \alpha_{1}(\|x\|) \leq V(t, x) \leq \alpha_{2}(\|x\|), \quad t \geq 0, \quad x \in \Delta, \\
& \underset{(1)}{C} D_{+}^{q} V\left(t, x ; 0, x_{0}\right) \leq-\alpha_{3}(\|x\|), \quad t>0, \quad x, x_{0} \in \Delta,
\end{aligned}
$$

where functions $\alpha_{i} \in C([0, \infty),[0, \infty)), i=1,2,3$, are strictly increasing and $\alpha_{i}(0)=0$. Then the equilibrium point $\bar{x}=0$ of system (1) is asymptotically stable.

\section{Main results}

In this section, we consider the multi-agent network that consists of $N$ agents and a leader. The dynamics of each agent, labeled $x_{i}, i=1,2, \ldots, N$, is given by the following equation:

$$
{ }_{0}^{C} D_{t}^{q} x_{i}(t)=f\left(t, x_{i}(t)\right)+u_{i}(t) \quad \text { for } i=1,2, \ldots, N, \quad x_{i}(0)=x_{0}^{i},
$$

where $q \in(0,1), x_{i}(t) \in \mathbb{R}$ is the state and $u_{i}(t)$ is the control input of the $i$ th agent at the moment $t, f \in C([0, T) \times \mathbb{R}, \mathbb{R}), T \leq \infty$, is a nonlinear function describing the intrinsic dynamics for the $i$ th agent. The virtual leader for multi-agent system (5) is an isolated 
agent described by

$$
{ }_{0}^{C} D_{t}^{q} x_{0}(t)=f\left(t, x_{0}(t)\right), \quad x_{0}(0)=x_{0}^{0},
$$

where $x_{0}$ is the state of the virtual leader. All agents and the leader share the same intrinsic dynamics.

In what follows, it is assumed that fractional-order system (5) and (6) has a unique solution for any initial values $\left(x_{0}^{0}, x_{0}^{1}, \ldots, x_{0}^{N}\right)^{T}$ (for the existence results see, for example, [31]).

Definition 2 ([3]) Multi-agent system (5) and (6) is said to achieve the leader-following consensus if a solution to (5) and (6) satisfies

$$
\lim _{t \rightarrow \infty}\left|x_{i}(t)-x_{0}(t)\right|=0, \quad i=1,2, \ldots, N
$$

for any initial values $x_{i}^{0} \in \mathbb{R}, i=0,1,2, \ldots, N$.

The control protocol is proposed as

$$
u_{i}(t)=-\beta(t)\left[\sum_{j=1}^{N} a_{i j}\left(x_{i}(t)-x_{j}(t)\right)+b_{i}\left(x_{i}(t)-x_{0}(t)\right)\right] \text {, }
$$

where $a_{i j}, i, j=1,2, \ldots, N$, is the $(i, j)$ th entry of the adjacency matrix $A \in \mathbb{R}^{N \times N}$ associated with the undirected graph $\mathcal{G}$ that describes the information exchange between agents; $b_{i}=1, i=1,2, \ldots, N$, if there exists the information flow from the virtual leader to agent $i$ and $b_{i}=0$ otherwise, $\beta \in C([0, \infty),(0, \infty))$ is the coupling strength function.

In what follows, $B=\operatorname{diag}\left\{b_{1}, b_{2}, \ldots, b_{N}\right\}$ and $H=L+B$, where matrix $L=\left[l_{i j}\right] \in \mathbb{R}^{N \times N}$ with $l_{i i}=\sum_{i \neq j} a_{i j}$ and $l_{i j}=-a_{i j}, i \neq j$, is called the graph Laplacian matrix induced by graph $\mathcal{G}$.

Let us denote by $y_{i}(t)=x_{i}(t)-x_{0}(t)$ the state error between the $i$ th agent and the leader. Then system (5) and (6) with $u_{i}$ given by (7) implies that

$$
\begin{aligned}
&{ }_{0}^{C} D_{t}^{q} y_{i}(t)= f\left(t, y_{i}(t)+x_{0}(t)\right)-f\left(t, x_{0}(t)\right) \\
&-\beta(t)\left[\sum_{j=1}^{N} a_{i j}\left(y_{i}(t)-y_{j}(t)\right)+b_{i} y_{i}(t)\right], \quad \text { for } t>0, \\
& y_{i}(0)=x_{0}^{i}-x_{0}^{0}, \quad i=1,2, \ldots, N .
\end{aligned}
$$

Let $\Lambda=P^{-1} H P$ be the Jordan canonical form of matrix $H$, where $P=\left\{p_{i j}\right\} \in \mathbb{R}^{N \times N}$ and its inverse $P^{-1}=\left\{\bar{p}_{i j}\right\} \in \mathbb{R}^{N \times N}$.

Set $z(t)=P^{-1} y(t)$, where $y(t)=\left(y_{1}(t), y_{2}(t), \ldots, y_{N}(t)\right)^{T}$. Then (8) can be rewritten in the vector form

$$
\begin{aligned}
& { }_{0}^{C} D_{t}^{q} z(t)=-\beta(t) \Lambda z(t)+P^{-1} F(t, P z(t)), \quad \text { for } t>0, \\
& z(0)=z_{0},
\end{aligned}
$$


where

$$
\begin{gathered}
F(t, y)=\left(f\left(t, y_{1}+x_{0}\right)-f\left(t, x_{0}\right), f\left(t, y_{2}+x_{0}\right)-f\left(t, x_{0}\right),\right. \\
\left.\ldots, f\left(t, y_{N}+x_{0}\right)-f\left(t, x_{0}\right)\right)^{T} \\
z_{0}=P^{-1} y_{0}, \quad y_{0}=\left(x_{0}^{1}-x_{0}^{0}, x_{0}^{2}-x_{0}^{0}, \ldots, x_{0}^{N}-x_{0}^{0}\right)^{T} .
\end{gathered}
$$

Remark 3 Observe that under an assumption $f(t, 0) \equiv 0$ for $t \geq 0, \bar{z}=0$ is an equilibrium point of system (9). On the other hand, if $\bar{z}=0$, then $y(t)=P \bar{z}=0$. Therefore, the fact that multi-agent system (5) and (6) with the proposed control law (7) achieves a leaderfollowing consensus is equivalent to the asymptotic stability of the equilibrium point $\bar{z}=0$ of system (9).

For $M \in \mathbb{R}^{N \times N}$, we consider the norm $\|M\|_{1}=\max _{1 \leq j \leq N} \sum_{i=1}^{N}\left|m_{i j}\right|$.

In the following two subsections, using the direct Lyapunov method, we will prove sufficient conditions for the leader-following consensus in system (5) and (6) with the control protocol given by (7). For this purpose, we assume that:

A1 $f(t, 0) \equiv 0$ for $t \geq 0$, i.e., $\bar{z}=0$ is an equilibrium point of system (9).

A2 Function $f$ is Lipschitz, i.e., there exists a function $L \in C([0, \infty),(0, \infty))$ such that $|f(t, z)-f(t, v)| \leq L(t)|z-v|$ for $z, v \in \mathbb{R}$ and $t \geq 0$.

\subsection{Intrinsic nonlinear dynamics with the bounded Lipschitz coefficient and time-varying coefficients in the control protocol}

Theorem 1 Assume that assumptions $\mathrm{A} 1$ and $\mathrm{A} 2$ hold. Additionally, let

(i) the Lipschitz coefficient $L \in C([0, \infty),(0, \infty))$ be bounded, i.e., there exists a constant $K>0$ such that $L(t) \leq K, t \geq 0$;

(ii) there exists a constant $\bar{\beta}>K \frac{\|P\|_{1}\left\|P^{-1}\right\|_{1}}{\min _{1 \leq i \leq N}\left\{\lambda_{i}\right\}}$ such that function $\beta$ satisfies the condition $\beta(t) \geq \bar{\beta}$ for all $t \geq 0$.

Then multi-agent system (5) and (6) with control law (7) achieves a leader-following consensus.

Proof Consider the quadratic function $V(t, x)=x^{T} x$ with $x \in \mathbb{R}^{N}$. Applying Lemma 1 to the solution $z(\cdot), t>0$, of system (9), we get

$$
\begin{aligned}
{ }_{0}^{C} D_{t}^{q} V(t, z(t)) \leq & 2 \sum_{i=1}^{N} z_{i}(t)_{0}^{C} D_{t}^{q} z_{i}(t) \\
= & 2 \sum_{i=1}^{N} z_{i}(t)\left[-\beta(t) \lambda_{i} z_{i}(t)\right. \\
& \left.+\sum_{j=1}^{N} \bar{p}_{i j}\left(f\left(t, \sum_{k=1}^{N} p_{j k} z_{k}(t)-x_{0}(t)\right)-f\left(t, x_{0}(t)\right)\right)\right] \\
\leq & 2 \sum_{i=1}^{N} z_{i}(t)\left[-\bar{\beta} \lambda_{i} z_{i}(t)+\sum_{j=1}^{N} \bar{p}_{i j}\left(L(t) \sum_{k=1}^{N} p_{j k}\left|z_{k}(t)\right|\right)\right] \\
\leq & -2 \bar{\beta} \sum_{i=1}^{N} \lambda_{i}\left(z_{i}(t)\right)^{2}+L(t) \sum_{i=1}^{N} \sum_{j=1}^{N} \bar{p}_{i j} \sum_{k=1}^{N} p_{j k} 2\left|z_{i}(t)\right|\left|z_{k}(t)\right|
\end{aligned}
$$




$$
\begin{aligned}
\leq & -2 \bar{\beta} \sum_{i=1}^{N} \lambda_{i}\left(z_{i}(t)\right)^{2}+L(t) \sum_{i=1}^{N} \sum_{j=1}^{N} \bar{p}_{i j} \sum_{k=1}^{N} p_{j k}\left(z_{i}(t)\right)^{2} \\
& +L(t) \sum_{i=1}^{N} \sum_{j=1}^{N} \bar{p}_{i j} \sum_{k=1}^{N} p_{j k}\left(z_{k}(t)\right)^{2} \\
\leq & -2\left(\bar{\beta} \min _{i}\left\{\lambda_{i}\right\}-K\left\|P^{-1}\right\|_{1}\|P\|_{1}\right) \sum_{i=1}^{N}\left(z_{i}(t)\right)^{2} .
\end{aligned}
$$

By assumptions A2 and (i), it follows that there exists a function $\alpha_{3} \in C([0, \infty),[0, \infty))$, $\alpha_{3}(0)=0$ such that ${ }_{0}^{C} D_{t}^{q} V(t, z(t)) \leq-\alpha_{3}(\|z\|)$. According to Lemma 2 , the equilibrium point $\bar{z}=0$ of system (9) is asymptotically stable. Therefore, by the substitution $Z(t)=P^{-1} Y(t)$, we get $\lim _{t \rightarrow \infty}\left|y_{i}(t)\right|=0$ or $\lim _{t \rightarrow \infty}\left|x_{i}(t)-x_{0}(t)\right|=0, i=1,2, \ldots, N$, which proves the claim.

Remark 4 If the Lipschitz coefficient in assumption A2 is a constant, i.e., $L(t) \equiv l$, then Theorem 1 is reduced to Theorem 11 from [13].

To illustrate the effectiveness of the sufficient conditions given in Theorem 1, we consider the modified example from [13].

Example 1 Let us consider a network of seven followers and a leader with matrices

$$
A=\left[\begin{array}{lllllll}
0 & 1 & 0 & 1 & 0 & 0 & 0 \\
1 & 0 & 1 & 0 & 1 & 0 & 0 \\
0 & 1 & 0 & 0 & 0 & 1 & 0 \\
1 & 0 & 0 & 0 & 0 & 0 & 1 \\
0 & 1 & 0 & 0 & 0 & 0 & 0 \\
0 & 0 & 1 & 0 & 0 & 0 & 0 \\
0 & 0 & 0 & 1 & 0 & 0 & 0
\end{array}\right], \quad B=\operatorname{diag}\{0,0,1,0,0,0,0\}
$$

Therefore, the model is

$$
\begin{aligned}
& { }_{0}^{C} D_{t}^{q} x_{0}(t)=e^{-t} \sin \left(x_{0}(t)\right), \\
& { }_{0}^{C} D_{t}^{q} x_{1}(t)=e^{-t} \sin \left(x_{1}(t)\right)-90\left(2 x_{1}(t)-x_{2}(t)-x_{4}(t)\right), \\
& { }_{0}^{C} D_{t}^{q} x_{2}(t)=e^{-t} \sin \left(x_{2}(t)\right)-90\left(3 x_{2}(t)-x_{1}(t)-x_{3}(t)-x_{5}(t)\right), \\
& { }_{0}^{C} D_{t}^{q} x_{3}(t)=e^{-t} \sin \left(x_{3}(t)\right)-90\left(3 x_{3}(t)-x_{2}(t)-x_{6}(t)-x_{0}(t)\right), \\
& { }_{0}^{C} D_{t}^{q} x_{4}(t)=e^{-t} \sin \left(x_{4}(t)\right)-90\left(2 x_{4}(t)-x_{1}(t)-x_{7}(t)\right), \\
& { }_{0}^{C} D_{t}^{q} x_{5}(t)=e^{-t} \sin \left(x_{5}(t)\right)-90\left(x_{5}(t)-x_{2}(t)\right), \\
& { }_{0}^{C} D_{t}^{q} x_{6}(t)=e^{-t} \sin \left(x_{6}(t)\right)-90\left(x_{6}(t)-x_{3}(t)\right), \\
& { }_{0}^{C} D_{t}^{q} x_{7}(t)=e^{-t} \sin \left(x_{7}(t)\right)-90\left(x_{7}(t)-x_{4}(t)\right), \\
& x_{i}(0)=x_{i}^{0}, \quad i=1,2, \ldots, 7 .
\end{aligned}
$$


Figure 1 The states of system (11) with initial values $(1,-5,8,-9,2,10,-3,0)^{T}$. The leader state is drawn in thick black and the states of followers are in blue

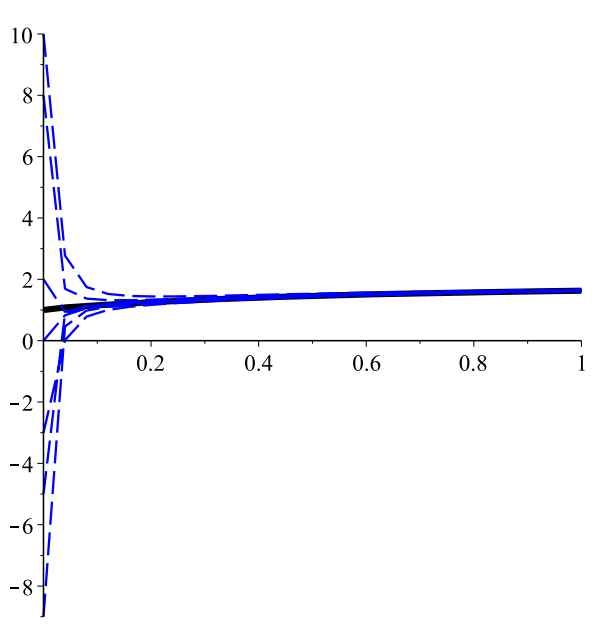

Figure 2 The states of system (11) with initial values $(18,-50,80,-19,20,60,-33,0)^{T}$. The leader state is drawn in thick black and the states of followers are in blue

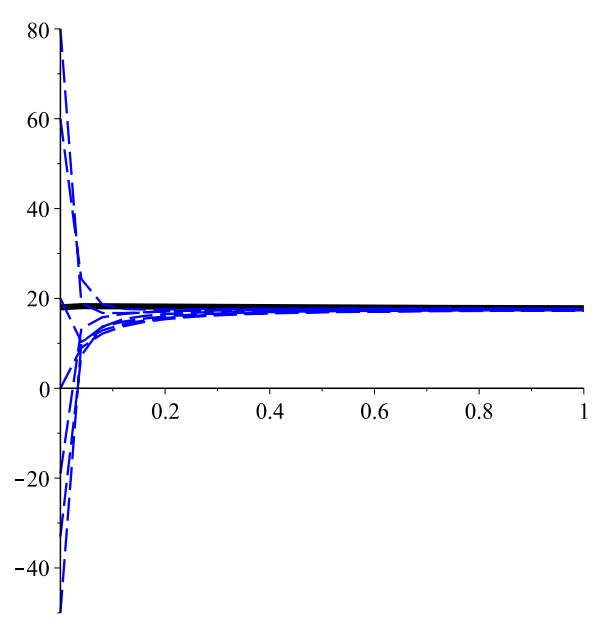

The eigenvalues of the matrix $H=L+B$ are $0.07,0.382,0.6767,1.4080,2.6180$, 3.2982, and 4.5472. We also have that $\|P\|_{1}=2.4835$ and $\left\|P^{-1}\right\|_{1}=2.3669$. The case with $f(t, z)=\frac{1}{6} \sin (z)$ was studied in [13]. Here we consider the nonlinear intrinsic dynamics of the virtual leader given by $f(t, z)=e^{-t} \sin (z)$. In this case, $L(t)=e^{-t}$ is a timevarying function, so the results of [13] could not be applied. However, if we choose $\bar{\beta}=90$ and $K=1$, then the conditions of Theorem 1 are satisfied and, what follows, the leader-following consensus is achieved. Figures 1 and 2 show the states of all agents with the nonlinear intrinsic dynamics of the virtual leader $f(t, z)=e^{-t} \sin (z)$ and two different initial values $(1,-5,8,-9,2,10,-3,0)^{T}$ and $(18,-50,80,-19,20,60,-33,0)^{T}$, respectively. It could be seen that the state of each follower asymptotically converges to the state of the leader, i.e., the leader-following consensus is achieved. Figures 1 and 2 show the states of all agents with initial values $(1,-5,8,-9,2,10,-3,0)^{T}$ and $(18,-50,80,-19,20,60,-33,0)^{T}$, respectively. When the nonlinear intrinsic dynamics of the virtual leader given by $f(t, z)=\sin (t) \sin (z)$ or the coupling strength function by $\beta(t)=90+\sin (t)$ assumptions of Theorem 1 still hold, and the leader-following consensus is achieved. Figures 3 and 4 show the states of all agents with initial values 
Figure 3 The states of all agents, from Example 1, with $f(t, z)=\sin (t) \sin (z)$. The leader state is drawn in thick black and the states of followers are in blue

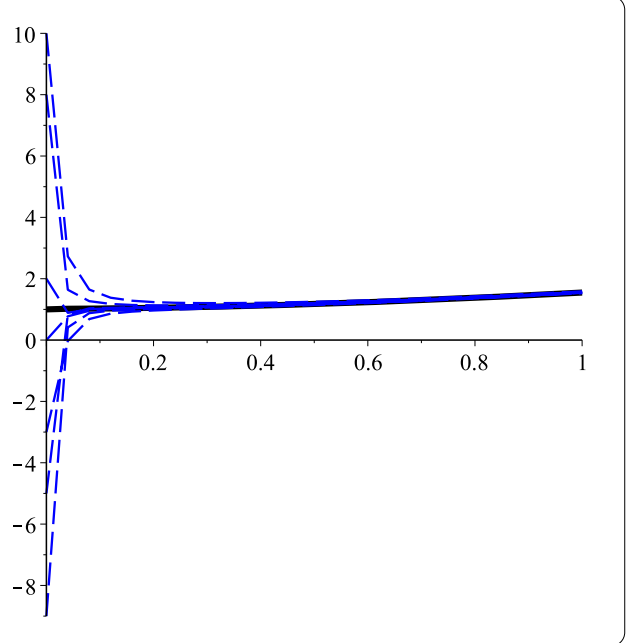

Figure 4 The states of all agents, from Example 1, with $\beta(t)=90+\sin (t)$. The leader state is drawn in thick black and the states of followers are in blue

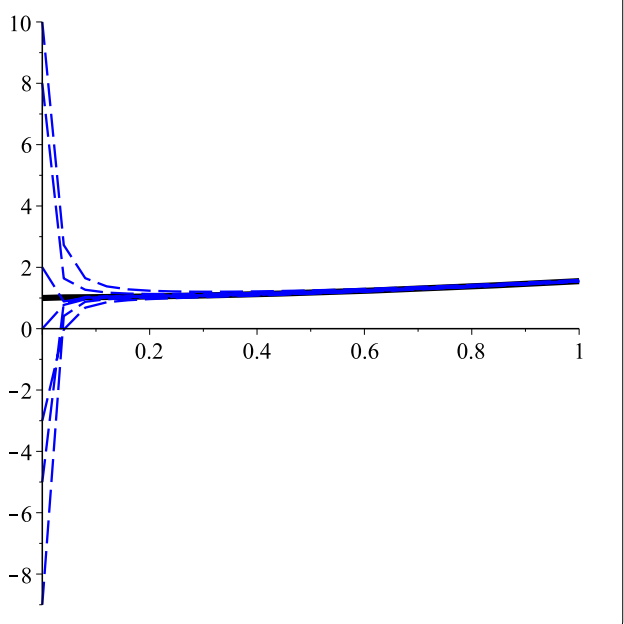

$(1,-5,8,-9,2,10,-3,0)^{T}$, and with $f(t, z)=\sin (t) \sin (z)$ and $\beta(t)=90+\sin (t)$, respectively. Apparently, the state of each follower asymptotically converges to the state of the leader, i.e., the leader-following consensus is achieved. We point out that the behavior of state trajectories is similar in spite of changing the coupling strength function $\beta$ and the initial values. Moreover, we observe very fast convergence to the consensus.

Remark 5 The value obtained for $\bar{\beta}$ is conservative. As shown in Figs. $1-4$, the bound for function $\beta$ can be in practice much smaller than theoretical one $\bar{\beta}$. The conservativeness of the bound is caused by: (1) the bound on the Caputo derivative of the Lyapunov function. In this respect, the approach which does not rely on the Lyapunov second method could provide a possible alternative; (2) the generality of the considered system. In this respect, the results for the case of the system with the constant coupling strength and the constant Lipschitz coefficient have been reported in [13]. 


\subsection{Intrinsic nonlinear dynamics with an unbounded Lipschitz coefficient and time-varying coefficients in the control protocol}

Theorem 2 Assume that assumptions A1 and A2 hold. Additionally, let there exist a function $g \in C([0, \infty),(0, \infty))$ such that

$$
L(t)\|P\|_{1}\left\|P^{-1}\right\|_{1}+\frac{{ }_{0}^{\mathrm{RL}} D_{t}^{q} g(t)}{g(t)}<2 \beta(t) \min _{1 \leq i \leq N} \lambda_{i}, \quad t \geq 0
$$

Then multi-agent system (5) and (6) with control law (7) achieves a leader-following consensus.

Proof Observe that using the quadratic function $V(t, x)=x^{T} x$, as in the proof of Theorem 1, we obtain inequality (10), which is not giving any bound of the Caputo fractional derivative ${ }_{0}^{C} D_{t}^{q} V(t, z(t))$ since now $L$ could be unbounded. Therefore, we consider the Lyapunov function $V(t, x)=g(t) x^{T} x, x \in \mathbb{R}^{N}$. By formula (3), for any point $z \in \mathbb{R}^{N}$, $z=\left(z_{1}, z_{2} \ldots, z_{N}\right)$, and $t>0$, the Dini fractional derivative for system (9) is

$$
\begin{aligned}
\mathcal{D}_{(5)}^{+} & V(t, z) \\
= & \limsup _{h \rightarrow 0} \frac{1}{h^{q}}\left[g(t) \sum_{i=1}^{N}\left(z_{i}\right)^{2}-\sum_{r=1}^{\left[\frac{t}{h}\right]}(-1)^{r+1}{ }_{q} C_{r} g(t-r h) \sum_{i=1}^{N}\left(z_{i}-h^{q} G_{i}(t, z)\right)^{2}\right] \\
= & \limsup _{h \rightarrow 0} \frac{1}{h^{q}}\left[g(t)\left(\sum_{i=1}^{N}\left(z_{i}\right)^{2}-\sum_{i=1}^{N}\left(z_{i}-h^{q} G_{i}(t, z)\right)^{2}\right)\right. \\
& \left.+\sum_{r=0}^{\left[\frac{t}{h}\right]}(-1)^{r}{ }_{q} C_{r} g(t-r h) \sum_{i=1}^{N}\left(z_{i}-h^{q} G_{i}(t, z)\right)^{2}\right] \\
= & \limsup _{h \rightarrow 0} \frac{1}{h^{q}}\left[h^{q} g(t) \sum_{i=1}^{N} G_{i}(t, z)\left(2 z_{i}-h^{q} G_{i}(t, z)\right)\right. \\
& \left.+\left(\sum_{i=1}^{N}\left(z_{i}-h^{q} G_{i}(t, z)\right)^{2}\right) \sum_{r=0}^{\left[\frac{t}{h}\right]}(-1)^{r}{ }_{q} C_{r} g(t-r h)\right] \\
= & \limsup _{h \rightarrow 0} g(t) \sum_{i=1}^{N} G_{i}(t, z)\left(2 z_{i}-h^{q} G_{i}(t, z)\right) \\
& +\left(\sum_{i=1}^{N} \limsup _{h \rightarrow 0}\left(z_{i}-h^{q} G_{i}(t, z)\right)^{2}\right) \limsup _{h \rightarrow 0} \frac{1}{h^{q}} \sum_{r=0}^{\left[\frac{t}{h}\right]}(-1)^{r}{ }_{q} C_{r} g(t-r h) \\
= & 2 g(t) \sum_{i=1}^{N} G_{i}(t, z)\left(z_{i}\right)+\left(\sum_{i=1}^{N}\left(z_{i}\right)^{2}\right){ }_{0}{ }_{0} D^{q} g(t),
\end{aligned}
$$

where $G_{i}(t, z)=-\beta(t) \lambda_{i} z_{i}+\sum_{j=1}^{N} \bar{p}_{i j}\left(f\left(t, \sum_{k=1}^{N} p_{j k} z_{k}-x_{0}\right)-f\left(t, x_{0}\right)\right)$. 
Hence

$$
\begin{aligned}
g(t) & \sum_{i=1}^{N} G_{i}(t, z) z_{i} \\
& =-\beta(t) g(t) \sum_{i=1}^{N} \lambda_{i}\left(z_{i}\right)^{2}+g(t) \sum_{j=1}^{N} \bar{p}_{i j}\left(f\left(t, \sum_{k=1}^{N} p_{j k} z_{k}-x_{0}(t)\right)-f\left(t, x_{0}\right)\right) z_{i} \\
& \leq\left(-\beta(t) g(t) \min _{1 \leq i \leq N}\left\{\lambda_{i}\right\}+g(t) L(t)\left\|P^{-1}\right\|_{1}\|P\|_{1}\right) \sum_{i=1}^{N}\left(z_{i}\right)^{2}
\end{aligned}
$$

Substituting (14) into (13) we get

$$
\begin{aligned}
& \mathcal{D}_{(5)}^{+} V(t, z) \\
& \quad \leq\left(-\beta(t) g(t) \min _{1 \leq i \leq N}\left\{\lambda_{i}\right\}+g(t) L(t)\left\|P^{-1}\right\|_{1}\|P\|_{1}+{ }_{0}^{\mathrm{RL}} D^{q} g(t)\right) \sum_{i=1}^{N}\left(z_{i}\right)^{2}, \quad t>0 .
\end{aligned}
$$

The claim follows by inequality (15), assumption (12), Remark 2, and Lemma 3.

Remark 6 Note that the main difficulty in the application of sufficient conditions provided by Theorem 2 is connected with obtaining an appropriate function $g$ in the Lyapunov function $V(t, x)=g(t) x^{T} x$.

The next example illustrates Theorem 2.

Example 2 Let us consider a network of seven followers and a leader, modeled by system (5) and (6) with control (7), where matrices $A$ and $B$ are defined in Example 1. Now, the nonlinear intrinsic dynamics of the leader is given by $f(t, z)=L(t) z$ with

$$
L(t)=\frac{1}{\|P\|_{1}\left\|P^{-1}\right\|_{1} \Gamma(1-q)} \frac{\Gamma(1-q) E_{q}\left(t^{q}\right)-1.1}{t^{q}\left(E_{q}\left(-t^{q}\right)+0.1\right)} \geq 0,
$$

where $\|P\|_{1}=2.4835$ and $\left\|P^{-1}\right\|_{1}=2.3669$ (see Example 1). Please note that the Lipschitz coefficient $L(\cdot)$ is not bounded (see Fig. 5).

Let us consider $\beta(t)=\frac{10 E_{q}\left(t^{q}\right)}{t^{q} \min _{1 \leq i>7} \lambda_{i}}$ and the Lyapunov function $V(t, z)=\left(E_{q}\left(-t^{q}\right)+\right.$ $0.1) \sum_{i=1}^{7}\left(z_{i}\right)^{2}$ for $t \geq 0, z \in \mathbb{R}^{7}$. Then inequality (12) reduces to

$$
\begin{aligned}
& L(t) g(t)\|P\|_{1}\left\|P^{-1}\right\|+{ }_{0}^{\mathrm{RL}} D^{q} g(t)-2 g(t) \beta(t) \min _{1 \leq i \leq 7} \lambda_{i} \\
& \quad=\frac{E_{q}\left(t^{q}\right)}{t^{q}}-\frac{1.1}{t^{q} \Gamma(1-q)}+{ }_{0}^{\mathrm{RL}} D^{q}\left(E_{q}\left(-t^{q}\right)+0.1\right)-2\left(E_{q}\left(-t^{q}\right)+0.1\right) \frac{10 E_{q}\left(t^{q}\right)}{t^{q}} \\
& =\frac{E_{q}\left(t^{q}\right)}{t^{q}}-\frac{1.1}{t^{q} \Gamma(1-q)}-E_{q}\left(-t^{q}\right)+\frac{1.1}{t^{q} \Gamma(1-q)}-2\left(E_{q}\left(-t^{q}\right)+0.1\right) \frac{10 E_{q}\left(t^{q}\right)}{t^{q}} \\
& \leq-\frac{E_{q}\left(t^{q}\right)}{t^{q}}\left(-1+10 E_{q}\left(-t^{q}\right)+1\right)=-10 \frac{E_{q}\left(t^{q}\right)}{t^{q}} E_{q}\left(-t^{q}\right)<0 .
\end{aligned}
$$

According to Theorem 2, the multi-agent system achieves the leader-following consensus, i.e., $\lim _{t \rightarrow \infty}\left|x_{i}(t)-x_{0}(t)\right|=0, i=1,2, \ldots, 7$. Figure 6 shows the states of all agents 


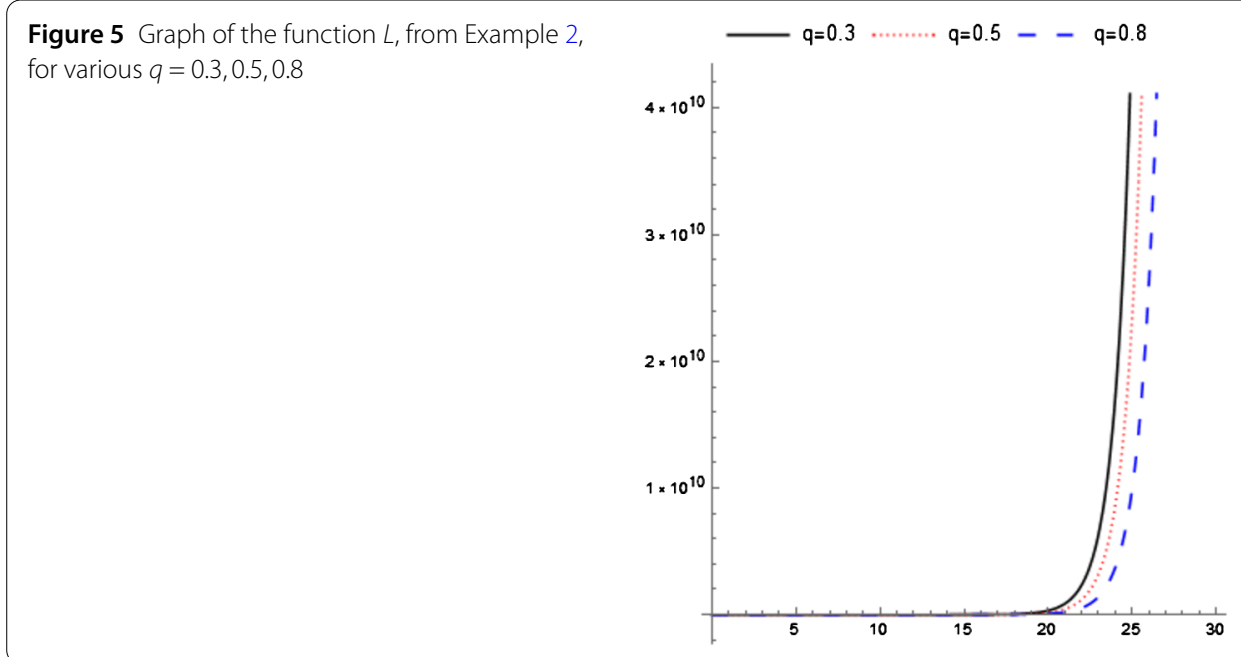

Figure 6 The states of all agents from Example 2 The leader state is drawn in thick black and the states of followers are in blue

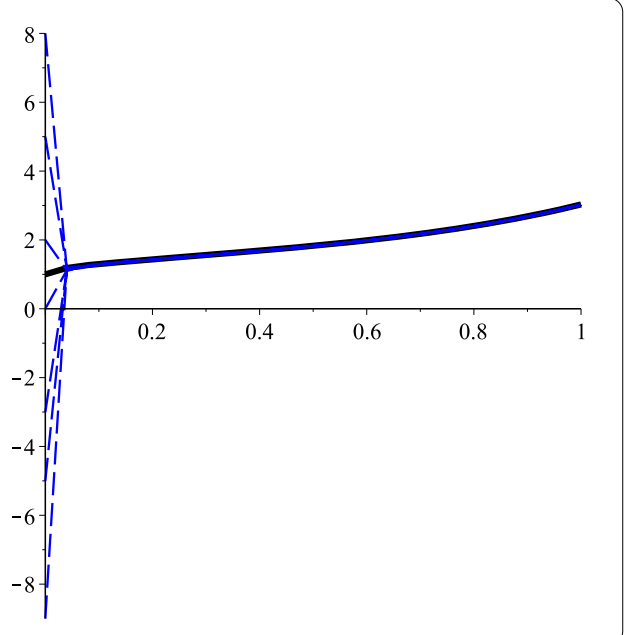

with initial values $(1,-5,8,-9,2,10,-3,0)^{T}$. It could be seen that the state of each follower asymptotically converges to the state of the leader.

\section{Conclusions}

The consensus problem is a key point in the dynamic multi-agent networks. In this paper, a leader-following consensus problem of Caputo fractional multi-agent systems was studied. Since asymptotic stability of the properly transformed system ensures the consensus in the considered model, stability analysis with the use of the second Lyapunov method was performed. We proposed a control protocol under which two types of examined multi-agent systems achieve a consensus. As a result, a set of sufficient conditions was derived to guarantee a consensus. Finally, numerical examples were presented to show the effectiveness of our theoretical results. Since many real-world phenomena are described by directed networks, such as the World Wide Web or mobile networks, as the future work the analysis of the case when the information exchange between agents is described by a directed graph can be considered. Investigating our approach to design a control law that guarantees the follower agents tracking their corresponding leaders in each subgroup (group consensus) [37] does also represent an interesting research venue. Other impor- 
tant directions of further investigation are scaled consensus with and without time delay [38-40] for fractional multi-agent systems problems. Those problems will be studied in subsequent papers.

\section{Acknowledgements}

The authors are thankful to the referees for helpful comments on this article.

\section{Funding}

R. Almeida was supported by Portuguese funds through the CIDMA—Center for Research and Development in Mathematics and Applications and the Portuguese Foundation for Science and Technology (FCT-Fundação para a Ciência e a Tecnologia), within project UID/MAT/04106/2019. A.B. Malinowska and E. Girejko were supported by the Polish National Science Center grant based on decision DEC-2014/15/B/ST7/05270. S. Hristova was supported by Fund FP19-FMI-002, University of Plovdiv "Paisii Hilendarski". Open access publishing was funded by the grant from the Polish National Science Center based on decision DEC-2014/15/B/ST7/05270.

\section{Availability of data and materials}

Not applicable.

\section{Competing interests}

The authors declare that there is no conflict of interests.

\section{Authors' contributions}

The authors read and approved the final manuscript.

\section{Author details}

'Center for Research and Development in Mathematics ad Applications, Department of Mathematics, University of Aveiro, Aveiro, Portugal. ${ }^{2}$ Faculty of Computer Science, Bialystok, University of Technology, Białystok, Poland. ${ }^{3}$ Faculty of Mathematics and Computer Science, University of Plovdiv Paisii Hilendarski, Plovdiv, Bulgaria.

\section{Publisher's Note}

Springer Nature remains neutral with regard to jurisdictional claims in published maps and institutional affiliations.

Received: 29 March 2019 Accepted: 11 July 2019 Published online: 24 July 2019

\section{References}

1. Jadbabaie, A., Lin, J., Morse, A.S.: Coordination of groups of mobile autonomous agents using nearest neighbor rules. IEEE Trans. Autom. Control 48, 988-1001 (2003)

2. Olfati-Saber, R., Murray, R.M.: Consensus problems in networks of agents with switching topology and time-delays. IEEE Trans. Autom. Control 49, 1520-1533 (2004)

3. Wei, N., Cheng, D.: Leader-following consensus of multi-agent systems under fixed and switching topologies. Syst. Control Lett. 59, 209-217 (2010)

4. Sakthivel, R., Sakthivel, R., Kaviarasan, B., Lee, H., Lim, Y.: Finite-time leaderless consensus of uncertain multi-agent systems against time-varying actuator faults. Neurocomputing 325, 159-171 (2019)

5. Mei, J., Ren, W., Chen, J.: Distributed consensus of second-order multi-agent systems with heterogeneous unknown inertias and control gains under a directed graph. IEEE Trans. Autom. Control 61, 2019-2034 (2016)

6. Song, Q., Cao, J., Yu, W.: Second-order leader-following consensus of nonlinear multi-agent systems via pinning control. Syst. Control Lett. 59, 553-562 (2010)

7. Yan, H., Shen, Y., Zhang, H., Shi, H.: Decentralized event-triggered consensus control for second-order multi-agent systems. Neurocomputing 133, 18-24 (2014)

8. Girejko, E., Mozyrska, D., Wyrwas, M.: Numerical analysis of behaviour of the Cucker-Smale type models with fractional operators. J. Comput. Appl. Math. 339, 111-123 (2018)

9. Mozyrska, D., Wyrwas, M.: Fractional discrete-time of Hegselmann-Krause's type consensus model with numerical simulations. Neurocomputing 216, 381-392 (2016)

10. Song, C., Cao, J., Liu, Y.: Robust consensus of fractional-order multi-agent systems with positive real uncertainty via second-order neighbors information. Neurocomputing 165, 293-299 (2015)

11. Almeida, R., Malinowska, A.B., Odzijewicz, T: Optimal leader-follower control for the fractional opinion formation model. J. Optim. Theory Appl. (2018). https://doi.org/10.1007/s10957-018-1363-9

12. Bai, J., Wen, G., Rahmani, A., Chu, X., Yu, Y.: Consensus with a reference state for fractional-order multi-agent systems, Int. J. Syst. Sci. 47, 222-234 (2015)

13. Ren, G., Yu, Y., Zhang, S.: Leader-following consensus of fractional nonlinear multiagent systems. Math. Probl. Eng 2015, $919757(2015)$

14. Yu, Z., Jiangn, $\mathrm{H}$., Hu, C.: Leader-following consensus of fractional-order multi-agent systems under fixed topology. Neurocomputing 149, 613-620 (2015)

15. Ahmad, W.M., El-Khazali, R.: Fractional-order dynamical models of love. Chaos Solitons Fractals 33, 1367-1375 (2007)

16. Song, L., XU, S.Y., Yang, J.Y.: Dynamical models of happiness with fractional order. Commun. Nonlinear Sci. Numer. Simul. 15, 616-628 (2010)

17. Lundstrom, B., Higgs, M., Spain, W.: Fractional differentiation by neocortical pyramidal neurons. Nat. Neurosci. 11 $1335-1342(2008)$ 
18. Li, C., Feng, G.: Delay-interval-dependent stability of recurrent neural networks with time-varying delay Neurocomputing 72, 1179-1183 (2009)

19. Li, G., Liu, H.: Stability analysis and synchronization for a class of fractional-order neural networks. Entropy 18,55 (2016)

20. Liu, H., Li, S., Wang, H., Huo, Y., Luo, J.: Adaptive synchronization for a class of uncertain fractional-order neural networks. Entropy 17, 7185-7200 (2015)

21. Wu, S., Li, C., Liao, X., Duan, S.: Exponential stability of impulsive discrete systems with time delay and applications in stochastic neural networks: a Razumikhin approach. Neurocomputing 82, 29-36 (2012)

22. Zhang, S., Yu, Y., Wang, H.: Mittag-Leffler stability of fractional-order Hopfield neutral networks. Nonlinear Anal. Hybrid Syst. 16, 104-121 (2015)

23. Hilfer, R.: Applications of Fractional Calculus in Physics. World Scientific, Singapore (2000)

24. Clarke, T., Narahari Achar, B.N., Hanneken, J.W.: Mittag-Leffler functions and transmission lines. J. Mol. Liq. 114, 159-163 (2004)

25. Sun, H., Abdelwahab, A., Onaral, B.: Linear approximation of transfer function with a pole of fractional power. IEEE Trans. Autom. Control 29, 441-444 (1984)

26. Petras, I., Vinagre, B.M.: Practical application of digital fractional-order controller to temperature control. Acta Montan. Slovaca 7, 131-137 (2002)

27. Aboelela, M.A.S., Ahmed, M.F., Dorrah, H.T.: Design of aerospace control systems using fractional PID controller. J. Advert. Res. 3, 225232 (2012)

28. Sun, H.G., Zhang, Y., Baleanu, D., Chen, W., Chen, Y.Q.: A new collection of real world applications of fractional calculus in science and engineering. Commun. Nonlinear Sci. Numer. Simul. 64, 213-231 (2018)

29. Shang, Y.: Fixed-time group consensus for multi-agent systems with non-linear dynamics and uncertainties. IET Control Theory Appl. 12, 395-404 (2018)

30. Shang, Y., Ye, Y.: Fixed-time group tracking control with unknown inherent nonlinear dynamics. IEEE Access 5 , 12833-12842 (2017)

31. Podlubny, I.: Fractional Differential Equations. Academic Press, San Diego (1999)

32. Duarte-Mermoud, M.A., Aguila-Camacho, N., Gallegos, J.A., Castro-Linares, R.: Using general quadratic Lyapunov functions to prove Lyapunov uniform stability for fractional order systems. Commun. Nonlinear Sci. Numer. Simul. 22, 650-659 (2015)

33. Li, Y., Chen, Y., Podlubny, I.: Stability of fractional-order nonlinear dynamic systems: Lyapunov direct method and generalized Mittag-Leffler stability. Comput. Math. Appl. 59, 1810-1821 (2010)

34. Devi, J.V., Mc Rae, F.A., Drici, Z.: Variational Lyapunov method for fractional differential equations. Comput. Math. Appl. 64, 2982-2989 (2012)

35. Li, Y., Chen, Y., Podlubny, I.: Mittag-Leffler stability of fractional order nonlinear dynamic systems. Automatica 45, 1965-1969 (2009)

36. Agarwal, R., O'Regan, D., Hristova, S.: Stability of Caputo fractional differential equations by Lyapunov functions. Appl. Math. 60, 653-676 (2015)

37. Shang, Y., Yamei, Y.: Leader-follower fixed-time group consensus control of multiagent systems under directed topology. Complexity 2017, 3465076 (2017)

38. Sakthivel, R., Kaviarasan, B., Ahn, C.K., Karimi, H.R.: Observer and stochastic faulty actuator-based reliable consensus protocol for multiagent system. IEEE Trans. Syst. Man Cybern. Syst. 48, 2383-2393 (2018)

39. Sakthivel, R., Kanakalakshmi, S., Kaviarasan, B., Ma, Y.K., Leelamani, A.: Finite-time consensus of input delayed multi-agent systems via non-fragile controller subject to switching topology. Neurocomputing 325, 225-233 (2019)

40. Shang, Y.: On the delayed scaled consensus problems. Appl. Sci. 7, 713 (2017)

\section{Submit your manuscript to a SpringerOpen ${ }^{\circ}$ journal and benefit from:}

- Convenient online submission

- Rigorous peer review

- Open access: articles freely available online

- High visibility within the field

- Retaining the copyright to your article

Submit your next manuscript at $\gg$ springeropen.com 